\title{
25th Anniversary Commemorative Issue
}

\author{
Deep Medhi ${ }^{1}$
}

Published online: 24 October 2017

(C) Springer Science+Business Media, LLC 2017

It seems like just yesterday that I received a flyer in my mailbox about a new journal on network management for which Manu Malek was going to be the editor-in-chief. This was in 1992. The first issue of the new journal, Journal of Network and Systems Management (JNSM), appeared in 1993. Because of my research interest that was in the scope of the journal, I submitted a paper at that time. My first paper in JNSM appeared in the 2nd issue in 1993. Since its inception, JNSM has continued to publish four issues a year. I was given the opportunity to serve as an associate editor with JNSM in 1996. Five years after that, Malek asked me to serve as one of the senior technical editors (STEs) starting in 2002.

After nearly two decades, Malek decided to step down from being the EiC. After eight years as an STE, I took over as the Editor-in-Chief officially in 2011, spending the second half of 2010 learning the ropes from Malek. My first editorial appeared in the first issue of 2011 [1]. I thank Malek for what I learned from him about this important responsibility. The journal's most recent Impact Factor is 1.588. Now, we're in 2017, and the journal that was just an infant yesterday has reached its 25 th year of publication. This is an appropriate moment to commemorate this significant milestone by having a special issue. I contacted a number of leading researchers in the network and system management community to contribute to this special commemorative issue. This special issue reflects the collection of papers received through this invited call. This collection can be grouped in four categories: (1) measurement, monitoring, and performance, (2) attacks and security management, (3) network functionalities and management: flexibility and directions, and (4) service management: experience and directions.

Deep Medhi

dmedhi@umkc.edu

1 Department of Computer Science Electrical Engineering, University of Missouri-Kansas City, 5110 Rockhill Road, Kansas City, MO 64110, USA 


\section{Measurement, Monitoring, and Performance}

An important problem in network monitoring and management is whether we can predict end-to-end performance by considering low-level network device statistics. Rolf Stadler, Rafael Pasquini, and Viktoria Fodor in "Learning from Network Device Statistics" take a statistical learning approach to show that this indeed can be accurately estimated. In particular, they were able to reduce the feature set of infrastructure statistics based on statistical learning that translates to estimating simply service-level metrics based on network device statistics - this is also a key connection between statistical learning and network performance prediction. Furthermore, this approach reduces the monitoring overhead through the collection of measurements simply from a path. They tested their approach for video streaming and on key-value store applications in a software-defined network environment.

Rafael Xavier, Lisandro Zambenedetti Granville, Bruno Volckaert, and Filip De Turck in "Elastic Resource Allocation Algorithms for Collaboration Applications" consider providing elasticity to real-time audio and video (A/V) collaboration applications in a cloud environment. The authors provided cloud provisioning algorithms and models in this setting. By using an extension of the CloudSim simulator, the benefits of this approach were illustrated.

\section{Attacks and Security Management}

In recent years, developing tools to analyze management data has become a paramount issue as networks and end devices face many issues including attacks. In "Flow-based Web Application Brute-force Attack \& Compromise Detection," Rick Hofstede, Mattijs Jonker, Anna Sperotto, and Aiko Pras address this problem for web applications. While in practice, where often a host based approach is used, the authors here have proposed a network based monitoring approach based on per-connection histograms of packet payload sizes in flow data that was shown to have a better ability to detect brute-force attacks against and compromises of web applications.

In "ROI-Driven Cyber Risk Mitigation Using Host Compliance and Network Configuration," Mohammed Noraden Alsaleh, Ehab Al-Shaer, and Ghaith Husari address the problem of security configuration, which requires a complex set of decision steps. They present a formal framework and new metrics to assess risks automatically and thereby, propose a cost-effective security consideration. Furthermore, their approach allows fine-grained access control.

\section{Network Functionalities and Management: Flexibility and Directions}

Data plane programmability has evolved from the early days of active networking to recent advances in software-defined networking. An important question that remains fully explored is: what challenges are faced to provide such functionalities from a network management perspective? In "Data Plane Programmability Beyond 
OpenFlow: Opportunities and Challenges for Network and Service Operations \& Management," Weverton Luis da Costa Cordeiro, Jonatas Adilson Marques, and Luciano Paschoal Gaspary start with an extensive survey in this topical area. Then, the authors ponder on challenges from a number of directions such as configuration management in a programmable data plane, and programmable security and dependability management. This exposition ends with a discussion on the future of data plane programmability with network management.

The 5th generation of mobile communication systems $(5 \mathrm{G})$ is imminent for us. Because of ultra-high bandwidth flexible services in $5 \mathrm{G}$ for applications relying on the cloud-radio access network (C-RAN), the access and backhaul transport network for $5 \mathrm{G}$ must have flexible on-demand service functionalities. In "Elastic Optical Networking for 5G Transport," Raouf Boutaba, Nashid Shahriar, and Siavash Fathi raise challenges and envision how an elastic optical transport network for 5G would need to be architected along with potential research directions.

\section{IT Service Management: Experience and Directions}

Yixin Diao and Larisa Shwartz's paper, "Building Automated Data Driven Systems for IT Service Management," delves into the IT service management space. In particular, they consider workload management, workflow management, and event management that form a complex interaction in IT service management. In light of challenges in these areas, they address autonomic systems that are made of a number of automated data driven methodologies from feedback controllers to machine learning techniques and show how this improves IT service management.

While there has been considerable progresses in service management over the past three decades, service management automation continues to face challenges in a continually changing world. Alexander Keller in his exposition, "Challenges and Directions in Service Management Automation," revisits what has changed over the years and provides possible directions on making advancements in service management automation.

Acknowledgements I would like to thank a number of people who helped me in getting this special issue out. First, I thank Luciano Paschoal Gaspary, Associate Managing Editor, for his input in putting together this commemorative issue. Melissa Fearon, Senior Editor of Computer Science and Engineering at Springer, agreed to this endeavor on a short notice. Priya Balamurugan, Journals Editorial Office (JOE) Assistant at Springer, ensured that everything is properly done by following up with me. I also thank Davis Light, Production Editor at Springer's Journal production, for ensuring that the production was completed in such a brief period so that we could bring out the special issue in a timely manner. Finally, I thank the reviewers who reviewed this collection of manuscripts on a very short notice.

\section{References}

1. Medhi, D.: Message from the New Editor-in-Chief. J. Netw. Syst. Manag. 19(1), 1-2 (2011) 